\title{
A literature review of leadership training and a novel conceptual model of leader-member exchange theory for new dentists
}

\author{
David O. Danesh \\ College of Dentistry, The Ohio State University, Columbus, Ohio, USA, and \\ Thomas P. Huber \\ College of Public Health, The Ohio State University, Columbus, Ohio, USA
}

LMX theory for new dentists

Received 28 May 2021 Revised 22 September 2021 Accepted 26 November 2021

\begin{abstract}
Purpose - The purpose of the paper is to describe the current state of leadership and leader-member exchange (LMX) theory in dentistry and develop a novel conceptual model of LMX to guide future research and highlight the importance of enhancing leadership training for new dentists.

Design/methodology/approach - A literature review exploring leadership in dentistry and LMX in dentistry was completed. The findings were analyzed with framework analysis to develop a novel conceptual model of LMX specific to dentistry.

Findings - LMX theory was applied to leadership in dentistry, including a focus on new dentists, senior dentists, other dental team members and the patient. A new conceptual model of the New Dentist LMX Quartet, which is unique and specific to new dentist teams, was developed.

Research limitations/implications - The study identifies the need for research in LMX in dentistry, contributes a new conceptual model for LMX theory and identifies future research.

Practical implications - Practitioners, policymakers and educators can utilize this information to explore concepts in leadership and improve training and dental practice.

Originality/value - No other studies specifically exploring LMX in dentistry for new dentists exist. The current literature review and conceptual paper begins the conversation on developing understanding of leadership in dentistry through further research.
\end{abstract}

Keywords Dentists, Health care, Higher education, Leaders, Teams, Leadership

Paper type Research paper

\section{Introduction}

Dentists play a key role in managing the oral health and overall health of patients. The dental team expands beyond the individual dentist with collaboration among and between dentists, dental specialists, dental hygienists, dental assistants, schedulers, financial coordinators, insurance specialists, receptionists and other clinic, support or administrative staff. Team member responsibilities may be fluid and oscillate between clinician, manager, supervisor, scheduler and other roles. Taichman et al. explain that the definition of leadership in dentistry includes not only "running a practice effectively, but a willingness to serve as a change agent and participate in the broader social, political and economic environment" (Taichman et al., 2009). However, a survey of general dentists found that $65 \%$ did not agree that "they had been well prepared by their pre-doctoral education to run an effective practice" (Taichman et al., 2014). Challenges and questions facing the future of dentistry can be answered with consideration of existing leadership theories. LMX theory examines the interactions within leader-member dyads while considering the quality of relationships, communication and awareness of biases to optimize interactions (Northouse, 2019). LMX theory can be used to analyze relationships of new dentists in dyads with others. This article focuses specifically on new dentists and reviews the literature on leadership training for new dentists and LMX in dentistry. A conceptual framework utilizing LMX theory called the New Dentist LMX Quartet 
is presented, which explores factors that contribute to dynamic relationship of new dentists as leaders and as members in dental teams. LMX Network theory is applied to develop vertical, horizontal and diagonal relationships of network dyads in dentistry. Directions for future research utilizing New Dentist LMX Quartet as well as opportunities to use the New Dentist LMX Quartet to integrate leadership development in practice for new dentists are presented.

\section{Leadership for dentists in training and new dentists}

USA dental practice is shifting from the individual solo dental practice model toward group practices with multiple dentists and dental specialists. An American Dental Association Health Policy Institute report found that about $65 \%$ of all private practice dentists in the USA were in solo practice in 1999 which decreased to about 50\% in 2019 (American Dental Association Health Policy Institute, 2021). USA dental school graduates can practice immediately after completing their dental degree and obtaining licensure, which presents a unique challenge for new dentists to transition into the responsibilities of a doctor. New dentists are increasingly practicing in group settings with experienced dentists and dental hygienists, assistants and staff members. New dentists may also even be treating patients who have been attending the practice for years. The challenges new dentists face emphasizes the importance of leadership skills in new dentists.

Leadership training in dental school curricula faces competing priorities with providing intensive training in basic sciences, pre-clinical skills, clinical experience and beyond, including barriers like curriculum crowding, cost and time commitment of students, staff and faculty (Victoroff et al., 2009; Niemchick et al., 2017). A 2010 survey of 54 USA dental schools found that almost three-quarters of dental schools that responded offered leadership training part of the current curriculum (Taichman and Parkinson, 2012). Leadership competences and skills in dental training should include communication, assertiveness, public speaking, the ability to listen to others, organizational skills, the ability to influence others, self-reflection, critical thinking, problem-solving, emotional intelligence, negotiation, conflict management, vision development, strategic planning, professionalism, ethics and social responsibility (Victoroff et al., 2008; Kalenderian et al., 2013). Strong leadership training in dental school can "inspire, encourage and enhance the development of the dental leaders of the future" (Victoroff et al., 2009). A 2007 survey of dental students at one dental school found that most students believed that "it is important for dentists to possess leadership skills and that leadership skills can be learned, and they expressed interest in participating in a leadership development program if such a program were offered" (Victoroff et al., 2008). Examples of leadership programs for dental students, dental residents, dental hygiene students and other dental school community members in the literature include as described in Table 1.

Leadership development programs for dental students have been implemented at dental schools, including Case School of Dental Medicine, University of Michigan and Harvard School of Dental Medicine through public speaking workshops, panel discussions, mentorship programs, team projects and sessions with leaders in dentistry (Taichman et al., 2009; Victoroff et al., 2009; Kalenderian et al., 2010). Surveys of dental students found that managing conflict was the weakest leadership skill reported (Kalenderian et al., 2010). Another survey found that most students found that learning leadership from mentors to be most effective method followed by small-group discussion, public speaking and other methods (Hammer and Nadershahi, 2011). Leadership programs can enhance studentfaculty engagement, encourage innovative collaboration, improve clinical efficiency, foster practice management knowledge, reduce medical errors and facilitate skills in communication, business, team-building, leadership and access to care (Taichman et al., 2009; Kalenderian et al., 2010). Investigators at the University of Michigan School of Dentistry 


Description
The University of Southern California School of Dentistry implemented an
elective leadership course that included faculty, dental students, dental residents
and staff in 2007
Implemented a pilot leadership program at Case Western Reserve University
School of Dental Medicine to increase dental student leadership knowledge and
skills
Piloted the Scholars Program in Dental Leadership (SPDL) for dental students
and dental hygiene students at the University of Michigan School of Dentistry
A dental student leadership course for all students was implemented in the
Harvard School of Dental Medicine curriculum
A 2010 survey established a foundation for a pilot leadership development
program for dental students at the University of the Pacific Arthur A. Dugoni
School of Dentistry
Harvard School of Dental Medicine implemented a post-graduate leadership
training course for dental residents in 2010
A follow-up survey for the Scholars Program in Dental Leadership (SPDL) at the
University of Michigan School of Dentistry positively influenced alumni
participant perceptions of leadership indicators and attitudes.

Authors (year)

Slavkin and Lawrence (2007)

Victoroff et al. (2009)

Taichman et al. (2009)

Kalenderian et al. (2010)

Hammer and Nadershahi (2011)

Skoulas and Kalenderian (2012)

Niemchick et al. (2017)
LMX theory for new dentists

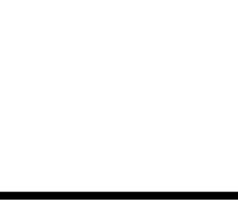
1 Literature review of
leadership training in new dentists

reported that alumni who participated in a dental school leadership development program had more positive views of community contribution, practice efficiency and evaluation of organized dentistry compared to alumni who did not participate in the leadership program (Niemchick et al., 2017).

As dentistry continues to change, new challenges present themselves at the individual level of a solo dental practice to the international scale of global oral health. These issues require multi-faceted solutions by future dentist-leaders. Given the importance of leadership in dentistry, a culture change in dental training is necessary and should include leadership skills and coursework early in dental training, augmenting clinical and technical training (Kalenderian et al., 2013). Beyond emphasizing the importance of leadership in dentistry and reporting the previous literature on leadership training in dentistry, developing a plan for future research of leadership in dentistry is critical. Taichman outlines several important questions for consideration regarding dental leadership. Does learning about leadership and definitions of leadership differ between dental students and practicing dentists (which includes a range of dentists from new graduates, dentists in residency training programs and experienced dentists) (Taichman, 2014)? What are the learning needs for dentists (Taichman, 2014)? What leadership skills are required for running an effective practice, functioning as a leader in the community and advocating from the local to national level and how do those skills differ (Taichman, 2014)? What is the most effective approach to integrate leadership training in dental training (Taichman, 2014)? In this article, some of these questions are addressed and potential solutions are offered by specifically examining new dentists in practice.

\section{Leader-member exchange theory}

LMX theory examines the interactions within a grouping called a dyad comprised of a leader and a member (Figure 1) while considering the importance of relationships, communication and awareness of biases to optimize interactions (Northouse, 2019). The dyad forms the basis of the characteristics and application of LMX theory. Figure 1 also describes the relationship between leaders and members, new dentists and dental team members and senior dentists and new dentists as examples of LMX dyads in dentistry. 


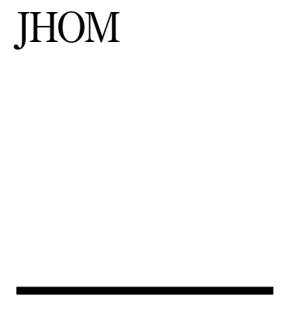

Figure 1.

Leader-member exchange dyads

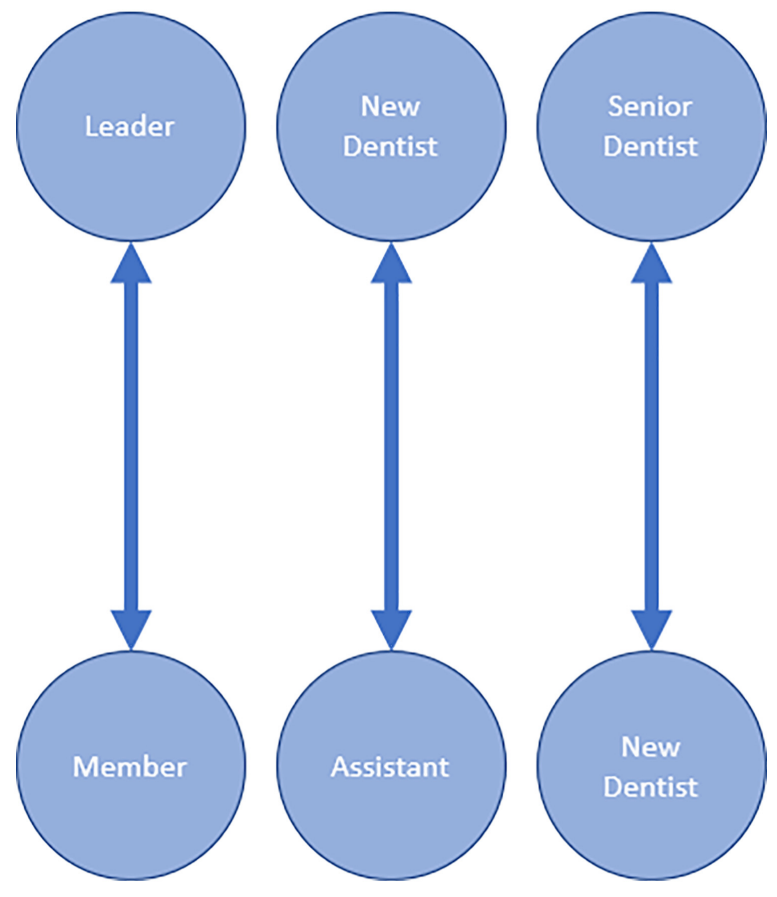

Vertical dyads were found to have two types of relationships between leaders and members described as in-groups and out-groups (Figure 2) (Northouse, 2019). In-groups are leadermember relationships that expand on formal negotiated role responsibilities through extra-roles beyond a formal employment contract (Northouse, 2019). This means that ingroup members will do tasks beyond their formal responsibilities for leaders, and leaders will reciprocate to do more for members of an in-group (Northouse, 2019). In contrast, out-group relationships are based specifically on a formal employment contract where members perform their defined roles without taking on new or additional tasks (Northouse, 2019). In-group members receive benefits such as "more information, influence, confidence and concern from leaders" compared to out-group members (Northouse, 2019). Figure 2 demonstrates how LMX dyads can include out-groups and in-groups for new dentist-assistant dyads and senior dentistnew dentist dyads.

Graen et al. further describe the characteristics LMX theory in a 1991 review (Graen and Uhl-Bien, 1991). Dyad relationships are further characterized into a progression of three relationship development phases: stranger, acquaintance and mature partnership (Figure 3) (Graen and Uhl-Bien, 1991). Some authors represent these phases of LMX relationship using a number scale, with 0 representing a stranger/low LMX relationship and +3 representing a mature/high LMX relationship. Figure 3 demonstrates how dyad relationships in dentistry can progress from a stranger/low LMX to an acquaintance phase to a mature/high LMX partnership of +3 .

Characteristics of leader-member dyads in the stranger relationship, or low LMX relationship, include "unidirectional downward influence, contractual behavior exchange, role-defined relations and loosely coupled goals" (Graen and Uhl-Bien, 1991). In the mature partnership relationship, or high LMX relationship, leaders can "exert considerable incremental influence" in dyads defined by "reciprocal influence, extra-contractual 

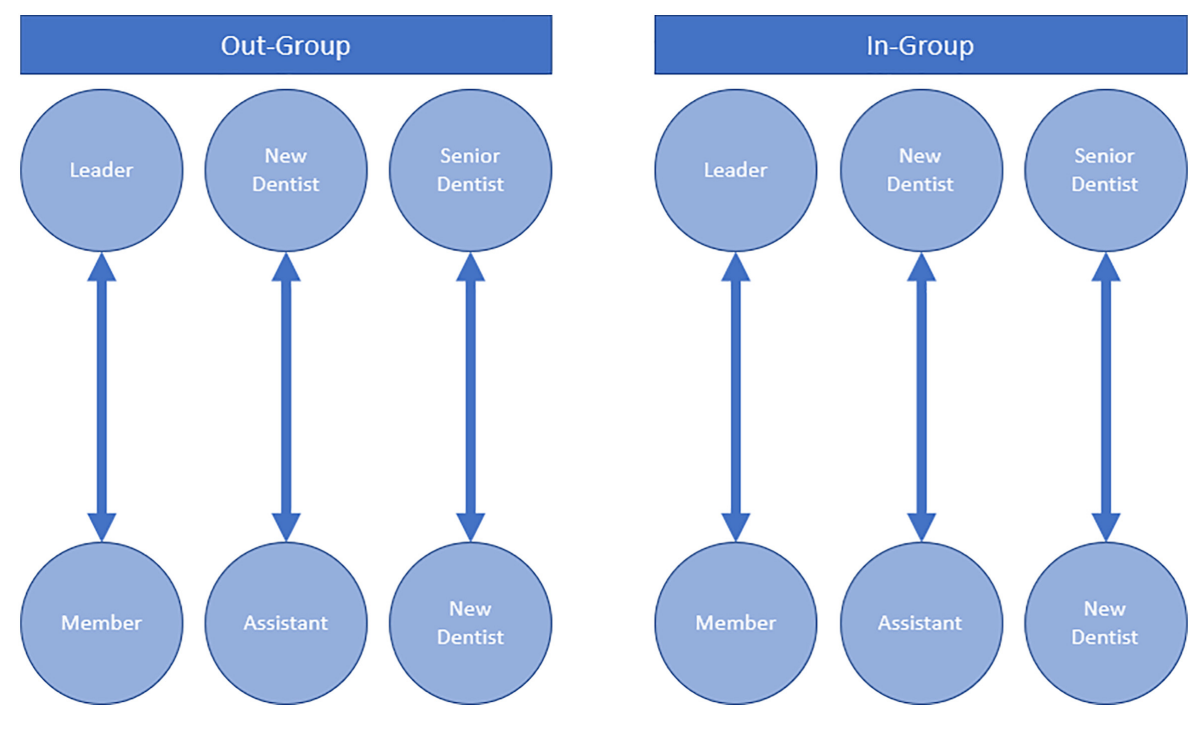

LMX theory for
new dentists

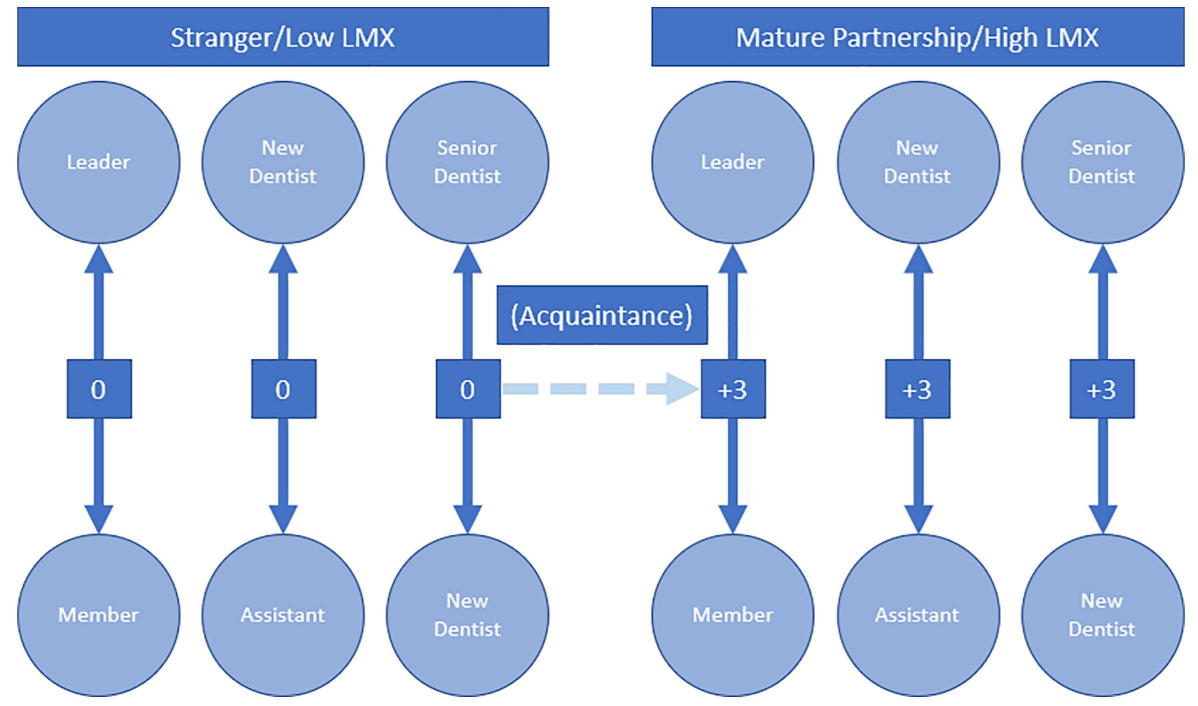

Figure 2.

Leader-member exchange in out-group and in-group

Figure 3.

Development of leader-member exchange dyads across relationships stages

behavior, mutual trust, respect and liking and internalization of common goals" (Graen and Uhl-Bien, 1991). The acquaintance relationship encompasses the continuum between the stranger and mature partnership relationship (Graen and Uhl-Bien, 1991).

The historical development LMX theory has been described by Graen et al. in a progression of four stages (Figure 4) (Graen and Uhl-Bien, 1991). Stage 1 of LMX theory includes the discovery and identification of dyads within work units as vertical dyad linkage; Stage 2 describes relationship characteristics and impact on organization outcomes, including in-groups and out-groups; Stage 3 further expands the theory and understanding of 
dyads to include partnership building and relationship analysis from stranger to acquaintance to mature partnership and, more recently, Stage 4 significantly broadens LMX relationships as assemblies of dyadic in large collectives at the group and network levels (Graen and Uhl-Bien, 1991).

\section{Applying LMX theory to address leadership challenges in dentistry}

When LMX theory is applied in the context of dentistry, individual dyad units also differ throughout stages of dental training. Dyads during dental school training include dental student-assistant, dental student-patient, dental student-faculty/senior dentist and dental student-dental student. Dyads after dental school training are similar but may occur in different contexts, which can include residency training, private practice, academic practice and public health among other settings. The dyads for a new dentist can include new dentistassistant, new dentist-patient, new dentist-senior dentist and new dentist-new dentist, where the new dentist can be a member or a leader. New dentist-senior dentist dyads can be complicated by other factors, such as the senior dentist may be the practice owner and direct supervisor of the new dentist, which introduces other power dynamics.

\section{Key findings}

Developing a conceptual framework of systems-level LMX theory in dentistry

Early LMX theory focused on dyad relationships within work groups and between leader and members. However, further work determined that leadership in larger and complex organizations include multiple vertical dyads with multiple leaders and multiple members "interacting collectivity" (Graen and Uhl-Bien, 1991). Stage 4 LMX theory "adopts a systemslevel perspective and pursues the question of how differentiated dyadic relationships combine together to form larger systems of network assemblies" and "constitute the leadership structure within the organization" (Graen and Uhl-Bien, 1991). LMX dyads "cut across work unit, functional, divisional and even organizational boundaries" with leadership relationships "among peers, teammates and across organizational levels and organizations" which may be formal or informal (Graen and Uhl-Bien, 1991). Stage 4 of LMX theory development maps an organizational leadership structure by examining task interdependencies and relationship quality at these multiple levels (Graen and Uhl-Bien, 1991). Applying Stage 4 of LMX theory to new dentist dyads in a network system of different leaders and members, including the patient, senior dentist and assistant can result in the development of a New Dentist LMX Quartet. Other new dentists can be considered in the quartet but will be excluded in this analysis. This paper introduces the idea of expanding and

Figure 4.

Development of leader-member exchange theory

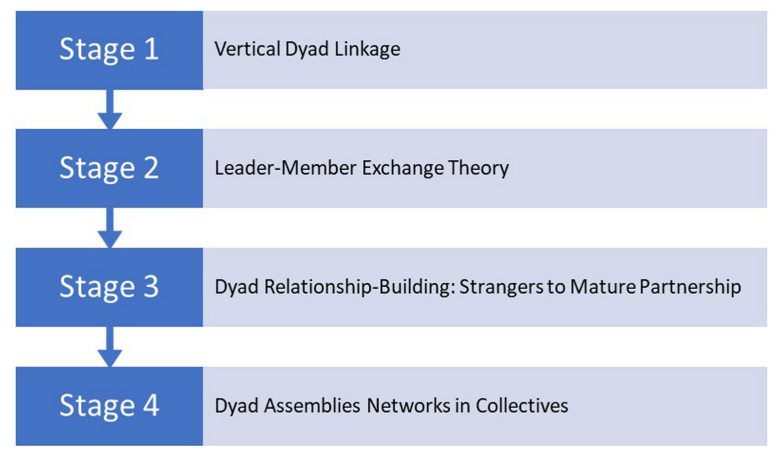


connecting dyads within dental practice with a focus on new dentists with stakeholders in a LMX Quartet (Figure 5). These dyads align with most directly with Stage 4 LMX theory of dyad assemblies in a network collective.

By developing the New Dentist LMX Quartet, independent and dependent factors that influence the dyad relationships for new dentists were identified. Independent factors are defined by the setting in which dental care is developed as opposed to the individual stakeholders forming the quartet. Independent factors include multiple cultures and multiple stakeholders. In-groups and out-groups influence quartet links but also are interactive among all links. Considering partnership building from stranger/low LMX to mature partnership/ high LMX relationship analysis adds another dimension to the New Dentist LMX Quartet. Furthermore, networks between new dentists and assistants, hygienists and other dental staff, senior dentists and patients provides additional consideration for in-group and outgroup influences and partnership influences on relationships. With these bidirectional networks applied in the context of Stage 4 of LMX theory, the New Dentist LMX Quartet model introduces the concept of diagonal and horizontal dyads in addition to vertical dyads described in Stage 1 of vertical dyad linkage.

\section{Discussion}

The New Dentist LMX Quartet allows for directed application of other studies of LMX to the context of new dentists and the vertical, horizontal and diagonal dyads with other leaders and members. The quartet and LMX theory can be utilized to understand and further explore leadership challenges faced by new dentists at the individual level of a solo dental practice to the international scale of global oral health. The quartet framework shows how multiple factors and networks interact, requiring multi-faceted training, skills and leadership perspective for dentists early in dental training. The development of the quartet also further contributes to the body of research on leadership and leadership training in dentistry. The quartet provides an additional definition of leadership for new dentists within the context of

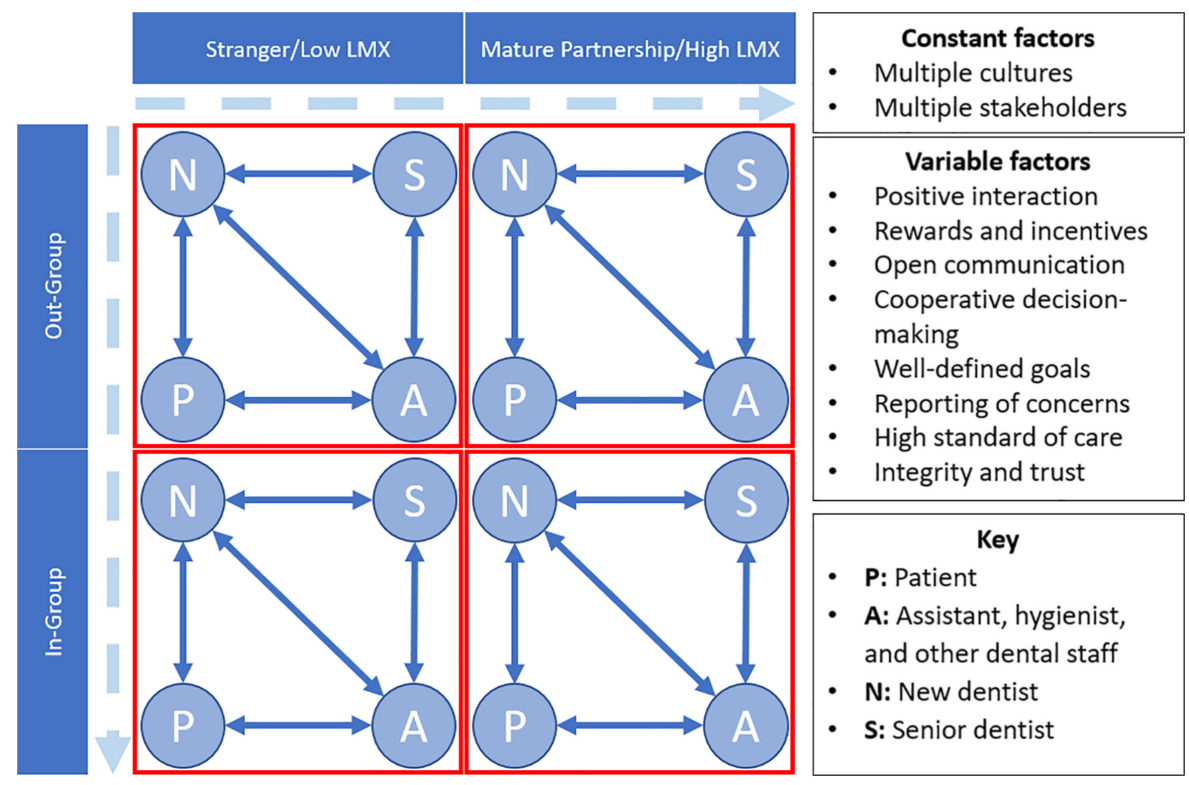

LMX theory for new dentists

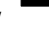


LMX theory, which is unique because a new dentist may be a leader or a member in multiple contexts. Furthermore, the relationship of the new dentist leaders differs if the follower is a patient or a dental team member. The new dentist-dental team member relationship is further complicated by different roles that a dental team member may have, such as an assistant, hygienist or other dental staff member. These relationships are also influenced by in-group or out-group status, as well as the maturity of the partnership. The quartet is also important in understanding the learning needs of new dentists, leadership skills required and the most effective approach to integrate this leadership training in practice.

A previous study found that applying LMX theory for training of medical resident physicians at a hospital in China under senior doctor teachers suggests that senior doctors should consider multiple cultures and multiple stakeholders in a single context, emphasize positive relationships and utilize incentives in training new medical residents (Deng et al., 2020). Considering the relationship between senior doctor teachers and new resident physicians is also called teacher-resident exchange (TRX) (Deng et al., 2020). Deng et al. apply stakeholder theory in that "the development of an organization cannot be separated from the involvement of various stakeholders," which means that stakeholder-related problems in TRX must consider relationships with multiple subjects (Deng et al., 2020). A residentoriented culture, which values residents as partners and assets to success, and a patientoriented culture, which puts the benefits of the customer first, were positively related to resident performance (Deng et al., 2020). When considering TRX between senior doctors and residents, TRX fully influenced resident training outcomes in resident-oriented culture and partially influenced resident-training outcomes in patient-orientated culture (Deng et al., 2020). Consistent with the New Dentist LMX Quartet, multiple stakeholders influence the training and development of new doctors and dentists, such as the patient and senior doctors. The New Dentist LMX Quartet also expands this relationship to include the strength of the relationship and additional stakeholders, like other dental team members. Another study explored leadership qualities of dental school faculty mentors that encouraged dental students to provide constructive suggestions and raise issues (Tang et al., 2019). Mentor leadership qualities included "openness for communication, cooperative decision making and well-defined goals" which led to trainees were significantly more likely to voice behaviors and raise issues (Tang et al., 2019). Effective communication forms the foundation of a strong LMX relationship, which can especially be applied in the context of new dentists and senior dentists.

\section{Stage 3 LMX: new dentist dyads from strangers to mature relationships}

Bunin et al. describe best practices utilizing LMX theory which may have saved a failed medical residency mentorship program across the stranger, acquaintance and mature partnership stages of mentor-protégé dyads (Bunin et al., 2020). These practices can be adapted in the context of the New Dentist LMX Quartet, as well as dependent and independent factors influencing the quartet, to address leadership challenges for new dentist dyads: leadership definitions and skills, differences in leadership learning approaches and development of practice management skills.

Within the stranger phase, new dentists should utilize cooperative decision-making to develop a formal educational plan with leaders and members to increase buy-in and engagement (Bunin et al., 2020). A formal education plan should account for differences in clinical experience of a new dentist, respecting their clinical autonomy while providing space for learning from clinical mistakes and growth. Defining a pre-written education plan to be used for reflection by new dentists reduces inconsistency and allows for objective review of new dentists rather than subjective assessment and areas to discuss. New dentists must clearly communicate their preferred learning approach through both verbal and written 
communication, as their learning occurs simultaneously with delivering patient care. Identifying and addressing dyads dissatisfaction and mismatch early is critical to success in this stage (Bunin et al., 2020). Assessing compatibility is a difficult process. New dentists should schedule formal and informal meetings to provide and receive feedback from all team members while also providing an outlet to review expectations, discuss bidirectional feedback and utilize conflict management (Bunin et al., 2020). Developing these leadership skills are important for new dentists, as they gain more responsibility in business and clinical management and to learn to run an effective practice as they will begin to hire and build their own teams.

In the acquaintance phase, new dentists' dyads should include continued education and critical evaluation as rules and boundaries of the relationship are navigated informally (Bunin et al., 2020). New dentists move beyond their individual education plan to mutual goals with team members and increase team building activities as the relationship matures are important aspects of this phase (Bunin et al., 2020). Creating consistent opportunities for formal and informal bidirectional feedback, both written and verbal, help with conflict management. New dentists benefit from this consistency due to the informal nature of the developing dyad. Other team members may have been operating in their respective dyads for years, while the new dentist may be a new addition to the team. At this point, some conflicts cannot be resolved leading to the need to terminate relationships of unsuccessful dyads (Bunin et al., 2020). The challenging process to terminate dyad relationships becomes more difficult when the new dentist joins a team that has been working together for an extended period.

During the mature partnership phase, new dentist dyads are strengthen based on communication, conflict management and positivity (Bunin et al., 2020). At this point, new dentists can continue effective learning approaches in a mature partnership while having the hindsight to eliminate ineffective learning approaches with strong dyads. Dependent factors that differ among different dyads contributed to unsuccessful dyads could include negative interaction and environment, lack of rewards or incentives, communication breakdown, illdefined goals, inability to report concerns, lack of commitment to quality and standard of care and a lack of integrity and trust. New dentists start to formulate a definition of leadership based on the dyad experience as well as the ability to apply these skills to other dyad relationships.

\section{Stage 4 LMX: application of the New Dentist LMX Quartet}

The LMX Quartet aligns most directly with Stage 4 LMX theory of dyad assemblies in a network collective. By analyzing individual LMX dyads, dentistry uniquely utilizes task interdependencies and relationship quality at multiple levels. Examining the LMX Quartet in the context of the literature identified independent and dependent factors that influence the dyad relationships for new dentists. The LMX Quartet also identified networks between a new dentist and stakeholders and provided consideration for in-group and out-group influences on relationships. Dependent factors are driven by the new dentist-leader and influenced by stakeholders and can include positive interactions, rewards and incentives, open communication, cooperative decision-making, well-defined goals, reporting of concerns and standard of care.

\section{Conclusion and highlights for decision-makers}

Challenges for the implementation of LMX include the perception of favoritism and special treatment between leaders and members. Significant gaps for research regarding leadership in dentistry were identified through this study and no previous research specifically explored LMX in dentistry. By considering vertical, horizontal and diagonal relationships of network 
dyads in dentistry, a new conceptual framework for LMX theory specific to dentistry was presented. The New Dentist LMX Quartet developed in this paper can be used for future research in explore case studies, quantitative analyses and qualitative interviews of new dentists. The New Dentist LMX Quartet allows for directed application of LMX theory to understand the learning needs, leadership skills and most effective training approaches of new dentists and opportunities to integrate leadership development in practice.

\section{References}

American Dental Association Health Policy Institute (2021), How Many Dentists Are in Solo Practice?, Health Policy Institute Infographic, Chicago, IL.

Bunin, J.L., Meyer, H.S. and Durning, S.J. (2020), "Could application of leader-member exchange theory have saved a residency mentorship program?", Perspectives on Medical Education, Vol. 9 No. 4, pp. 264-267, doi: 10.1007/s40037-020-00584-2.

Deng, G., Zhao, D., Lio, J., Ma, X., Liang, L. and Feng, C. (2020), "Linking hospital culture to the training performance of residents: the roles of leader-member exchange and transactional leadership style", Journal of Evaluation in Clinical Practice, Vol. 26 No. 1, pp. 92-100, doi: 10. 1111/jep.13204.

Graen, G. and Uhl-Bien, M. (1991), "The transformation of professionals into self-managing and partially self-designing contributors: toward a theory of leadership-making”, Journal of Management Systems, Vol. 3 No. 3, pp. 25-39.

Hammer, D.A. and Nadershahi, N.A. (2011), "Assessing the demand and preferred format of a student leadership development program at Pacific", Journal of Dental Education, Vol. 75 No. 8, pp. 1044-1052.

Kalenderian, E., Skoulas, A., Timothé, P. and Friedland, B. (2010), "Integrating leadership into a practice management curriculum for dental students", Journal of Dental Education, Vol. 74 No. 5, pp. 464-471, doi: 10.1002/j.0022-0337.2010.74.5.tb04892.x.

Kalenderian, E., Taichman, R., Skoulas, A., Nadershahi, N. and Victoroff, K. (2013), "Developing the next generation of leaders in oral health", Journal of Dental Education, Vol. 77 No. 11, pp. 1508-1514, doi: 10.1002/j.0022-0337.2013.77.11.tb05627.x.

Niemchick, A., Delgado, J., Taichman, R. and Inglehart, M. (2017), "Dental students', alumni, and dentists' perspectives on leadership: impact of the scholars program in dental leadership", Journal of Dental Education, Vol. 81 No. 1, pp. 44-53, doi: 10.1002/j.0022-0337.2017.81.1. tb06246.x.

Northouse, P.G. (2019), Leadership: Theory and Practice, 8th ed., Sage Publications, Thousand Oaks.

Skoulas, A. and Kalenderian, E. (2012), "Leadership training for postdoctoral dental students", Journal of Dental Education, Vol. 76 No. 9, pp. 1156-1166, available at: https:/proxy.lib.ohio-state.edu/login? url=http://search.ebscohost.com/login.aspx?direct $=$ true \&db=ehh\&AN=79992585\&site=edslive\&scope $=$ site.

Slavkin, H. and Lawrence, L. (2007), "Incorporating leadership knowledge and skills into the dental education community", Journal of Dental Education, Vol. 71 No. 6, pp. 708-712, doi: 10.1002/j. 0022-0337.2007.71.6.tb04328.x.

Taichman, L.S., Taichman, R.S. and Inglehart, M.R. (2014), "Dentists' leadership-related educational experiences, attitudes, and past and current behavior", Journal of Dental Education, Vol. 78 No. 6, pp. 876-885, doi: 10.1002/j.0022-0337.2014.78.6.tb05741.x.

Taichman, R.S. (2014), "Expanding the scholarship on leadership training in dental education", Journal of Dental Education, Vol. 78 No. 6, pp. 811-812, doi: 10.1002/j.0022-0337.2014.78.6.tb05733.x.

Taichman, R.S., Green, T.G. and Polverini, P.J. (2009), "Creation of a scholars program in dental leadership (SPDL) for dental and dental hygiene students", Journal of Dental Education, Vol. 73 No. 10, pp. 1139-1143. 
Taichman, R.S. and Parkinson, J.W. (2012), "Where is leadership training being taught in U.S. dental schools?”, Journal of Dental Education, Vol. 76 No. 6, pp. 713-720, doi: 10.1002/j.0022-0337.2012. 76.6.tb05305.x.

LMX theory for new dentists

Tang, W.C., Kingsley, K. and Reinke, R. (2019), "Impact of collaborative leadership in dental school team clinics", Journal of Dental Education, Vol. 83 No. 12, pp. 1436-1444, doi: 10.21815/JDE. 019.155 .

Victoroff, K.Z., Schneider, K. and Perry, C. (2008), "Leadership development for dental students: what do students think?", Journal of Dental Education, Vol. 72 No. 9, pp. 982-988, doi: 10.1002/j.00220337.2008.72.9.tb04572.x.

Victoroff, K.Z., Schneider, K. and Perry, C. (2009), "Tomorrow's leaders, starting today: a pilot leadership development program for dental students", Journal of Dental Education, Vol. 73 No. 3, p. 311, available at: https://proxy.lib.ohio-state.edu/login?url=http://search.ebscohost. com/login.aspx?direct $=$ true $\& \mathrm{db}=$ edsbl\&AN $=\mathrm{RN} 247357086 \&$ site $=$ eds-live\&scope $=$ site .

\section{Corresponding author}

David O. Danesh can be contacted at: davidodanesh@gmail.com

For instructions on how to order reprints of this article, please visit our website: 